\title{
Fay-Herriot Model-Based Prediction Alternatives for Estimating Households with Emigrated Members
}

\author{
Jairo Fúquene-Patiño ${ }^{1}$, César Cristancho ${ }^{2}$, Mariana Ospina ${ }^{2}$, and \\ Domingo Morales Gonzalez ${ }^{3}$
}

\begin{abstract}
This article proposes a new methodology for estimating the proportions of households that had experience of international migration at the municipal level in Colombia. The Colombian National Statistical Office usually produces estimations of internal migration based on the results of population censuses, but there is a lack of disaggregated information about the main small areas of origin of the population that emigrates from Colombia. The proposed methodology uses frequentist and Bayesian approaches based on a Fay-Herriot model and is illustrated by one example with a dependent variable from the Demographic and Health Survey 2015 and covariables available from the population census 2005. The proposed alternative produces proportion estimates that are consistent with sample sizes and the main internal immigration trends in Colombia. Additionally, the estimated coefficients of variation are lower than $20 \%$ for municipalities for both frequentist and Bayesian approaches and large demographically-relevant capital cities and therefore estimates may be considered to be reliable. Finally, we illustrate how the proposed alternative leads to important reductions of the estimated coefficients of variations for the areas with very small sample sizes.
\end{abstract}

Key words: Small area estimation; international migration; Fay-Herriot model; coefficient of variation; direct estimator; model-based estimator; hierarchical Bayes prediction.

\section{Introduction}

In Colombia (as well as in other developing countries) there is a lack of demographic sources of information related to migration. The indicators of internal migration and international immigration are usually based on questions on population censuses that ask about the place of residence five years earlier for people who live in Colombia. On other hand, for measuring international emigration there are two options: (1) the administrative records of international flights and migration checkpoints and (2) the estimations of international migrations based on population censuses of other countries. In both cases, the statistics involved are crucial for estimating the total number of emigrants, but they are not adequate sources of information for detailed estimation of subnational areas of origin of

\footnotetext{
${ }^{1}$ UC Davis, Department of Statistics, Davis, California, 95616-5270, U.S.A. Email: jafuquenepatino@ ucdavis.edu

${ }^{2}$ National Department of Statistics, Population projections division, Bogota, Colombia. Emails: cacristanchof@dane.gov.co and mfospinab@dane.gov.co

${ }^{3}$ The Miguel Hernández University of Elche (UMH), Centro de Investigación Operativa, Departamento de Estadistica, Matemáticas, Avenida de la Universidad s/n ELCHE, 03202, Spain. Email: d.morales@umh.es Acknowledgments: JFP was supported by the CAMPOS Initiative - UC Davis. DM was supported by the Spanish grant PGC2018-096840-B-I00. We thank Brenda Betancourt (University of Florida) for her insights on the Bayesian approach. We also thank the associate editor and three referees for their valuable comments.
} 
international emigration. Furthermore, one problem of Option (2) is that, unfortunately, not all countries have population censuses in the same year.

The registered data by Migracion-Colombia (2015) reflect the upward trends in emigration over the years, but it is necessary to have estimations of the main places of origin of international emigration at subnational level because this information is relevant for elaborating small area migration projections. We should take into account that changes in the demographic characteristics of origin areas are affected by international emigration (Edmonston and Michalowski 2004). In Colombia there are more than 1000 municipalities organized in 33 departments and although we expect the main cities to be the most important places of origin of international emigration. However this is not true in all cases and it is important to elaborate a hierarchy of the most important places of origin of international migration flows.

There is a need of a methodology that integrates the available sources of information to identify the main small areas of emigration in Colombia, because we identify that there is a marked contrast between the growing need for disaggregated data at a local level for planning purposes and the lack of adequate sources, tools and research with the level of detail required. Despite the fact that internal migration has a greater impact than international migration in terms of population growth and demographic change in the majority of municipalities, there is a growing need to investigate the effects of international migration, as it has a significant impact on the demographic dynamics of some of the areas of interest. See Smith et al. (2013) for a practical guide on state and local population projections.

This article introduces a statistical procedure to estimate, at the municipal level, the proportion of households that have at least one usual member who is living abroad. For ease of exposition, this indicator is called "proportion of households with at least one usual member living abroad" (PHMLA). This is a quantity of interest to analyze the demographic evolution of the municipalities of Colombia and, as mentioned by Edmonston and Michalowski (2004), no country has made a serious effort to fully exploit the results obtained for this indicator, but it remains a serious possibility. Thus, the proposed methodology is based on the exploration of relatively new information that, so far, has not been used in detail.

An important source of information for estimating PHMLAs at the municipal level is the population census carried out in 2005 by the National Administrative Department of Statistics (DANE). Another recent information source is the DHS, Demographic and Health Survey of 2015 (Profamilia 2015) in which it is possible to measure, at the departmental level, the proportion of households that have at least one usual member living abroad. The Colombian DHS includes a set of questions related to international emigration. This information source is very well-known and several developing nations use its results to monitor not only population health, but also a wide range of subjects including migration, fertility, mortality, family planning, reproductive health, and HIV/AIDS.

It is possible to make estimations of PHMLAs for 2015 at the municipal level with support from corresponding PHMLAs obtained in the 2005 census, which can display considerable bias if taken as linear trends over the years. For this reason, it is essential for the country to be able to estimate PHMLAs at the municipal level with greater precision. A methodological proposal for mapping PHMLAs at the municipal level would enable 
estimations in intercensal periods and, additionally, would help to update estimations on the level and structure of population dynamics in Colombia. Likewise, a migration study at the municipal level would help to illustrate the health, education and employment requirements of the new population.

The goal of this article is to explore for the first time (to the best of our knowledge), the use of small area estimation (SAE) theory to estimate PHMLAs in intercensal periods at the municipal level. Using the Colombian demographic context as a reference, this article also establishes whether the estimates of PHMLAs based on the proposed methodology are reliable and in keeping with population dynamics. Therefore, this work proposes an alternative methodological framework to estimate the PHMLA at the municipal level in Colombia.

Statistical models have helped researchers to estimate migration flows between regions or countries. For example, Willekens (2008) applied unit-level models to calculate the probability that individuals in the population will migrate. At the aggregated level, Plane (1982), Raymer (2007) and Smith at al. (2010) proposed gravity, entropy maximization and log-linear models to estimate aggregate migration flows between countries and Raymer and Rogers (2007) incorporated spatial dependence and socio-economic similarities between origin and destination countries. These approaches cannot be directly applied to the Colombian case because of the unavailability of appropriate data sources. In fact, they are not designed to consider the problem of using survey data to estimate international migration in regions where the amount available information is small. As Raymer (2010) pointed out, the case of international migration is more complicated because of the inconsistencies in migrant definitions and data collection methods between countries.

The sampling and inference techniques for finite populations can be used to estimate demographic quantities, like counts and proportions of people displaying an event under study. As the PHMLAs is a proportion, one possibility is to model the dichotomic variables indicating wheter or not a household has emigrated members. Hobza and Morales (2016, 2018) followed this approach for estimating small area poverty proportions and they proposed empirical best predictors based on unit-level logit mixed models. Unfortunately, their approaches require the availability of auxiliary variables for all the population units and, therefore, it is not applicable to the Colombian data.

Alternatively, the area-level model approach to SAE relies on previous calculations of direct estimators of PHMLAs and their variances. These direct estimates are entered into a Fay-Herriot $(\mathrm{FH})$ model that enables the calculation of empirical best linear unbiased predictors (EBLUP) of PHMLAs at the municipal level. Rao and Molina (2015)e gave a complete introduction to the theory and practice of SAE.

The Fay-Herriot model is a linear mixed model proposed by Fay and Herriot (1979) to estimate the average per capita income in small areas in the United States. Esteban et al. (2012) and Morales et al. (2015), Benavent and Morales (2016) and Burgard et al. (2020a, b) extended this methodology to the estimation of small area poverty proportions under temporal, bivariate and measurement error Fay-Herriot models respectively, but they did not give applications to demography or to population dynamics. Therefore this article investigates the potential applications of the area-level SAE methodology to the estimation of demographic indicators. 
The rest of this article is organized into three sections. Section 2 introduces the FayHerriot model and presents the frequentist and Bayesian predictors of PHMLAs. This section also provides an adequate modeling of the estimated design-based variances of the direct estimators. Section 3 applies the methodology of Section 2 to estimate the PHMLA in municipalities of Colombia. Additionally, Section 3 carries out a model diagnosis along with a comparison with traditional estimators. Finally, Section 4 presents some conclusions related to the obtained results.

\section{Migration Estimation Under the Fay-Herriot Model}

Consider a finite population of size $N$, where the units are households. For each household $j$, the variable of interest is $y_{j}=1$ if at least one person who is a usual member of the household $j$ currently lives abroad, and it is $y_{j}=0$ if no one in the household $j$ currently lives abroad. Assume that the population is divided into small areas labelled by $d=1, \ldots, D$. In our case, they are the municipalities of Colombia. Let $s$ be a sample extracted at random from the population according to a given sample design. The population and the sample are denoted by $U=\cup_{d=1}^{D} U_{d}$ and $s=\cup_{d=1}^{D} s_{d}$, respectively. Let $N_{d}$ and $n_{d}$, be the sizes of $U_{d}$ and $s_{d}, d=1, \ldots, D$. The objective is to estimate the PHMLAs per municipality, $\bar{Y}_{d}=N_{d}^{-1} \sum_{j \in U_{d}} y_{j}$, with its respective coefficient of variation. The direct estimator of $\bar{Y}_{d}$, proposed by Hájek (1971), and the corresponding estimated coefficient of variation are

$$
\hat{\bar{Y}}_{d}^{d i r}=\frac{1}{\hat{N}_{d}} \sum_{j \in s_{d}} w_{j} y_{j}, \quad \mathrm{c} \hat{\mathrm{v}}^{d i r}\left(\hat{\bar{Y}}_{d}^{d i r}\right)=\frac{1}{\hat{\bar{Y}}_{d}^{d i r}} \sqrt{\frac{1}{\hat{N}_{d}^{2}} \sum_{j \in s_{d}} w_{j}\left(w_{j}-1\right)\left(y_{j}-\hat{\bar{Y}}_{d}^{d i r}\right)^{2}},
$$

where $\hat{N}_{d}=\sum_{j \in s_{d}} w_{j}$ is the direct estimator of the population size of municipality $d$ and $w_{j}$ is the sample weight of household $j$. The sample weights of the DHS2015 survey are obtained from a complex sampling design in four stages: (1) municipalities, (2) urban blocks and rural areas, (3) segments and, (4) people. The quartiles of the sample sizes of the $D=282$ municipalities represented in the DHS2015 survey are $n^{(0)}=7, n^{(1)}=47$, $n^{(2)}=89, n^{(3)}=170, n^{(4)}=3,069$. Therefore, estimating municipality indicators is a SAE problem.

The direct estimators are approximately unbiased for $\bar{Y}_{d}, d=1, \ldots, D$, but they are not usually precise for estimating small areas parameters. Because of the small sample sizes, they lead to considerably high coefficients of variation ( $>20 \%)$. See Särndal et al. (1992) for more details about inference in finite population and properties of direct estimators. Small area estimators might be based on unit-level or area-level models. Under the arealevel approach, a model is fitted to the aggregated data $\left(\hat{\bar{Y}}_{d}^{d i r}, x_{d}\right), d=1, \ldots, D$, where $x_{d}$ is a row vector that contains the values of $p$ auxiliary variables. The use of area- level SAE procedures has the disadvantage of using auxiliary variables aggregated at the municipality level, with the possible loss of information with respect to the data aggregated at a lower level (for example, blocks, segments or people). However, this disadvantage is offset by using all the auxiliary variables available at the municipal level. This fact also prevents the restriction of estimators based on unit-level models that must have the same auxiliary variables in the survey file and in the external administrative records. 


\subsection{The Fay-Herriot Model}

Fay and Herriot (1979) introduced an area-level linear mixed model for SAE. The FayHerriot model for estimating the municipal proportion of households, with at least one member who has emigrated abroad, can be expressed in the matrix form

$$
y=X \beta+u+e,
$$

where $\boldsymbol{y}=\left(\hat{\bar{Y}}_{1}^{d i r}, \ldots, \hat{\bar{Y}}_{D}^{d i r}\right)^{\prime}$ is the vector that contains the direct proportion estimates, $\boldsymbol{X}=\operatorname{col}_{1 \leq d \leq D}\left(\boldsymbol{x}_{d}\right)$ is the $D \times p$ matrix containing the values of $p$ auxiliary variables, $\beta=\left(\beta_{1}, \ldots, \beta D\right)^{\prime}$ is the vector of regression parameters. Concerning the random effects and errors, the model assumes that $\boldsymbol{u}=\left(u_{1}, \ldots, u_{D}\right)^{\prime} \sim N\left(\mathbf{0}, \sigma_{d}^{2} I_{D}\right)$ and $\boldsymbol{e}=\left(e_{1}, \ldots, e_{D}\right)^{\prime} \sim N(\boldsymbol{0}$, $\left.\boldsymbol{V}_{\mathrm{e}}\right)$ are independent, where $\boldsymbol{V}_{\mathrm{e}}=\operatorname{diag}_{1 \leq d \leq D}\left(\sigma_{d}^{2}\right)$ and the variances $\sigma_{d}^{2}$ are assumed to be known. In practice, the error variances $\sigma_{d}^{2}$ can be substituted by estimates $\hat{\sigma}_{d}^{2}$ calculated from unit-level data. In any case, the Fay-Herriot treats these estimates as known constants.

When applying the Fay-Herriot model, the underlying idea is that the direct estimators of the proportions are not accurate, given that the municipality sample sizes are small. For this reason, the model improves the estimates by taking auxiliary information from the explanatory variables $\boldsymbol{x}_{d}$, including the variability between municipalities through the random effectv $u_{d}$ and taking into account the random sampling error $e_{d}$ derived from using a direct estimator as model target variable.

\subsection{Frequentist Methods for the Fay-Herriot Model}

There are several frequentist methods for estimating the parameters of Equation (2), such as maximum likelihood (ML), residual maximum likelihood (REML) or method of moments. This article applies the REML method to calculate an estimator $\sigma_{u}^{2}$ of the random effect variance. The REML estimator of $\hat{\sigma}_{u}^{2}$ has lower bias than its $\mathrm{ML}$ counterpart. Furthermore, the implementation of the REML method avoids potential negative variance estimates, such as in the case of the method of moments. See, for example, Jiang (2007) for an introduction to the ML and REML estimators in linear mixed models and the consequent calculation algorithms. The REML estimator of the vector of regression parameters and the EBLUP of the random effects are

$$
\hat{\beta}=\left(X^{\prime} \hat{V}^{-1} X\right)^{-1} X^{\prime} \hat{V}^{-1} y, \quad \hat{u}_{d}=\frac{\hat{\sigma}_{u}^{2}}{\hat{\sigma}_{u}^{2}+\hat{\sigma}_{d}^{2}}\left(\hat{\bar{Y}}_{d}^{d i r}-x_{d} \hat{\beta}\right), \quad d=1, \ldots, D .
$$

where $\hat{V}=\operatorname{diag}\left(\hat{\sigma}_{u}^{2}+\hat{\sigma}_{d}^{2}, \ldots, \hat{\sigma}_{u}^{2}+\hat{\sigma}_{D}^{2}\right)$.

The REML estimator $\hat{\beta}$ is a weighted least squared estimator. The EBLUPs of the random effects can be interpreted as weighted residuals that take into account the variability of the direct estimators that is not explained by the auxiliary variables. Under the Fay-Herriot Equation (2), the EBLUP of $\bar{Y}_{d}$ is

$$
\hat{\bar{Y}}_{d}^{F H}=\frac{\hat{\sigma}_{u}^{2}}{\hat{\sigma}_{u}^{2}+\hat{\sigma}_{d}^{2}} \hat{\bar{Y}}_{d}^{d i r}+\frac{\hat{\sigma}_{d}^{2}}{\hat{\sigma}_{u}^{2}+\hat{\sigma}_{d}^{2}} x_{d} \hat{\beta}, \quad d=1, \ldots, D .
$$

The EBLUP (4) is a weighted average of the estimate produced by the direct estimator and the values predicted by Equation (2). If the sample size per municipality $n_{d}$ is large, it 
is understood that $\hat{\sigma}_{d}^{2} \approx 0$ and therefore the EBLUP and the direct estimator are approximately equal, that is, $\hat{\bar{Y}}_{d}^{F H} \approx \hat{\bar{Y}}_{d}^{d i r}$. Conversely, if in the calculation of the direct estimator there is a small sample size per municipality, then $\hat{\sigma}_{d}^{2}>\hat{\sigma}_{u}^{2}$ and therefore the EBLUP is approximately equal to the values predicted in the Fay-Herriot model, that is, $\hat{\bar{Y}}_{d}^{F H} \approx x_{d} \hat{\beta}$. Chapter 4 of Rao and Molina (2015) reviews the basic properties of the FayHerriot model and the related applications to SAE problems.

For calculating the Equation (4), it is necessary to estimate the $\sigma_{d}^{2}$ variances. Using unitlevel data and applying the Equation (1), it is possible to obtain direct estimates, $\operatorname{vâ}\left(\hat{\bar{Y}}_{d}^{d i r}\right)$, of the variance of the direct estimator $\hat{\bar{Y}}_{d}^{d i r}$. However, these estimates are not precise since the sample sizes of the municipalities are generally very small. An alternative estimation procedure uses auxiliary data and applies a log-linear model for the variance estimates of direct estimators. This is the method of the Generalized Variance Function (GVF), which is described, for example, in Schall (1991). The GVF method fits the model

$$
\log \left(\operatorname{vâr}\left(\hat{\bar{Y}}_{d}^{d i r}\right)\right)=x_{d}^{v} \beta^{v}+\varepsilon_{d}^{v}, \quad d=1, \ldots, D,
$$

where $\varepsilon_{d}^{d i r} \sim N\left(0, \sigma_{\varepsilon}^{2}\right), d=1, \ldots, D$, are independent. In order to estimate $\sigma_{d}^{2}$, the predicted values are obtained from Equation (5) using the following formula

$$
\hat{\boldsymbol{\sigma}}_{d}^{2}=\exp \left\{\hat{\boldsymbol{\sigma}}_{\varepsilon}^{2} / 2\right\} \exp \left\{x_{d}^{v} \hat{\boldsymbol{\beta}}^{v}\right\}, \quad d=1, \ldots, D,
$$

where $\exp \left\{\hat{\sigma}_{\varepsilon}^{2} / 2\right\}$ is the bias-correction term in log-normal regression. This article selects the auxiliary variables contained in the vector $x_{d}^{v}$ from the direct estimates of PHMLAs, $\hat{\bar{Y}}_{d}^{d i r}$, the sample sizes $n_{d}$ and the interaction between them. For improving the predictive capacity of the GVF Equation (5), the included covariates can be raised to an exponent, resolving potential problems of heteroscedasticity. In practice, when the correction term is not used, the GVF method tends to underestimate the variances.

To estimate the coefficient of variation of the EBLUP, $\hat{\bar{Y}}_{d}^{F H}$, we approximate the mean squared error (MSE). Prasad and Rao (1990) gave the MSE estimator

$$
\operatorname{MSE}\left(\hat{\bar{Y}}_{d}^{F H}\right)=g_{1}\left(\hat{\boldsymbol{\sigma}}_{u}^{2}\right)+g_{2}\left(\hat{\boldsymbol{\sigma}}_{u}^{2}\right)+2 g_{3}\left(\hat{\boldsymbol{\sigma}}_{u}^{2}\right)
$$

where

$$
\begin{aligned}
& g_{1}\left(\hat{\sigma}_{u}^{2}\right)=\frac{\hat{\sigma}_{u}^{2} \hat{\sigma}_{d}^{2}}{\hat{\sigma}_{u}^{2}+\hat{\sigma}_{d}^{2}}, \quad g_{2}\left(\hat{\sigma}_{u}^{2}\right)=\frac{\hat{\sigma}_{d}^{4}}{\left(\hat{\sigma}_{u}^{2}+\hat{\sigma}_{d}^{2}\right)^{2}}\left(X^{\prime} V^{-1} X\right)^{-1} x_{d}^{\prime} \\
& g_{3}\left(\hat{\sigma}_{u}^{2}\right)=\frac{\hat{\sigma}_{d}^{4}}{\left(\hat{\sigma}_{u}^{2}+\hat{\sigma}_{d}^{2}\right)^{3}} \operatorname{avar}\left(\hat{\sigma}_{u}^{2}\right)
\end{aligned}
$$

with $g_{3}\left(\hat{\sigma}_{u}^{2}\right)$ given by Datta and Lahiri (2000) and where the asymptotic variance is

$$
\operatorname{avar}\left(\hat{\sigma}_{u}^{2}\right)=2\left(\sum_{d=1}^{d} 1 /\left(\hat{\sigma}_{u}^{2}+\hat{\sigma}_{d}^{2}\right)^{2}\right)^{-1}
$$




\subsection{Bayesian Methods for the Fay-Herriot Model}

Hierarchical Bayes models represent another alternative to fit the Fay-Herriot (Datta et al. 2011). The Bayes estimates for PHMLAs are obtained by considering the Fay-Herriot Equation (2) in hierarchical form as follows

$$
\begin{aligned}
& y=\theta+e, \\
& \theta=X \beta+u,
\end{aligned}
$$

where $\boldsymbol{\theta}=\left(\theta_{1}, \ldots, \theta_{D}\right)^{\prime}$ is the vector of random e?ects. In this work, we use a suitable prior distribution under $\boldsymbol{u}$ and $\boldsymbol{\theta}$ given by $\pi(\boldsymbol{u}, \boldsymbol{\theta}) \propto 1$. For the posterior inference of the parameter vector $(\boldsymbol{\theta}, \boldsymbol{\beta}, \boldsymbol{u})$, we use samples $s=1, \ldots, S$ from the full conditionals of the Gibbs sampling scheme given in Rao and Molina (2015) and denoted by $\left(\boldsymbol{\theta}^{(s)}, \boldsymbol{\beta}^{(\mathrm{s})}, \boldsymbol{u}^{(\mathrm{s})}\right)$. The Hierarchical Bayes (HB) predictor of the PHMLA and the corresponding variance for the small area $d$ are obtained from the Rao-Blackwell estimator as follows

$$
\hat{\bar{\theta}}_{d}^{H B}=\frac{1}{S} \sum_{s=1}^{S} \hat{\theta}_{d}^{(s) H B} ; \quad V\left(\hat{\bar{\theta}}_{d}^{H B}\right)=\frac{1}{S} \sum_{s=1}^{S} g_{1}\left(\hat{\boldsymbol{\sigma}}_{u}^{(s) 2}\right)+\frac{1}{S-1} \sum_{s=1}^{S}\left(\hat{\theta}_{d}^{(s) H B}-\hat{\bar{\theta}}_{d}^{H B}\right)^{2},
$$

where

$$
\hat{\theta}_{d}^{(s) H D}=\frac{\hat{\sigma}_{u}^{(s) 2}}{\hat{\sigma}_{u}^{(s) 2}+\hat{\sigma}_{d}^{(s) 2}} \hat{\bar{Y}}_{d}^{d i r}+\frac{\hat{\sigma}_{d}^{(s) 2}}{\hat{\sigma}_{u}^{(s) 2}+\hat{\sigma}_{d}^{(s) 2}} x_{d} \hat{\beta}^{(s)}, \quad d=1, \ldots, D .
$$

\section{Estimating International Emigration from Small Areas in Colombia}

This section fits a Fay-Herriot model to DHS2015 data and calculates the EBLUP of the selected indicator of international emigration at the municipal level. Subsection 3.1 applies the GVF method to estimate the variances of the direct estimators of PHMLAs. The final variance estimates are the predicted values of a selected log-linear regression model. Subsection 3.2 presents the comparisons between the EBLUPs and the direct estimators of PHMLAs and the corresponding diagnosis of the fitted Fay-Herriot model.

\subsection{Modeling the Variance of the Direct Estimator}

As mentioned in Section 2, in order to improve the application of the Fay-Herriot model a GVF estimation of the variances of the direct estimators is carried out. Since in some municipalities the direct estimate and its variance are equal to zero, a constant $\delta>0$ is added to the variance of the direct estimators in the GVF Equation (5) to prevent numerical errors and/or heteroscedasticity in the residuals. In practice, there are different possibilities for the selection of the value $\delta$. In this work, we take 5 as the average of the direct estimates of the design-based variances, which is $\delta=0.0056$.

Once the Equation (5) is fitted to data, we undo the transformation $\log \left(\operatorname{vâr}\left(\hat{\bar{Y}}_{d}^{d i r}\right)+\delta\right)$ without applying the bias-correction term $\exp \left\{\hat{\sigma}_{\varepsilon}^{2} / 2\right\}$. In fact, this term does not correct the bias if we sum $\delta$ to $\operatorname{vâr}\left(\hat{\bar{Y}}_{d}^{d i r}\right)$. This approach has two competing consequences. By summing $\delta$, the GVF method tends to over-estimate the variances because some zero 
variances are substituted by $\delta$. By not applying a bias correction term, the GVF method tends to underestimate the variances. Figures 1 and 2 gives information about the goodness-of-fit of the GVF model to data and about the quality of the variance predictions.

For the GVF Equation (5), the selected explicative variables and parameters are

$$
\begin{aligned}
& x_{d}^{v}=\left(1, \hat{\bar{Y}}_{d}^{d i r},\left(\hat{\bar{Y}}_{d}^{d i r}\right)^{1 / 2}, n_{d}, n_{d}^{1 / 2},\left(\hat{\bar{Y}}_{d}^{d i r} n_{d}\right)^{1 / 2}\right), \\
& \hat{\beta}^{v}=\left(\beta_{1}^{v}, \beta_{2}^{v}, \beta_{3}^{v}, \beta_{4}^{v}, \beta_{5}^{v}, \beta_{6}^{v}\right)^{\prime} .
\end{aligned}
$$

We propose the explanatory variable $\left(\hat{\bar{Y}}_{d}^{d i r}\right)^{1 / 2}$ because it has the same scale of values as $\hat{\bar{Y}}_{d}^{d i r}$. The selection of the variables of the log-linear model is made through the AIC,
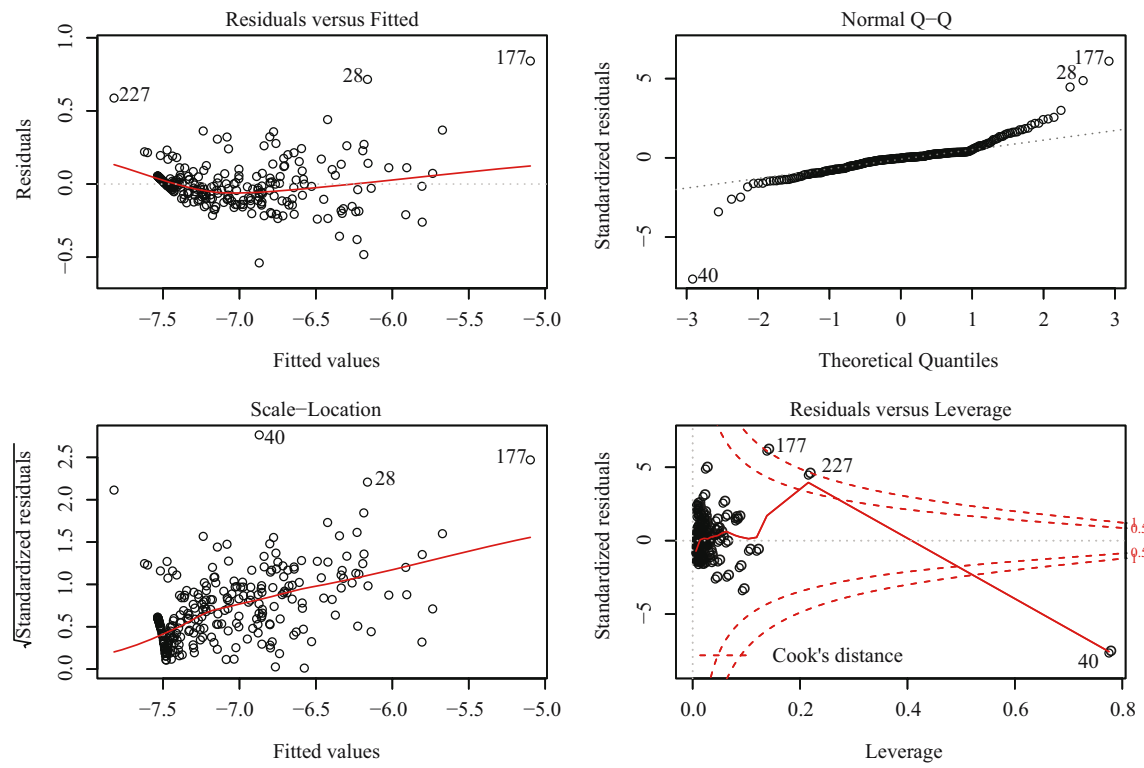

Fig. 1. Residuals of the selected GVF model.
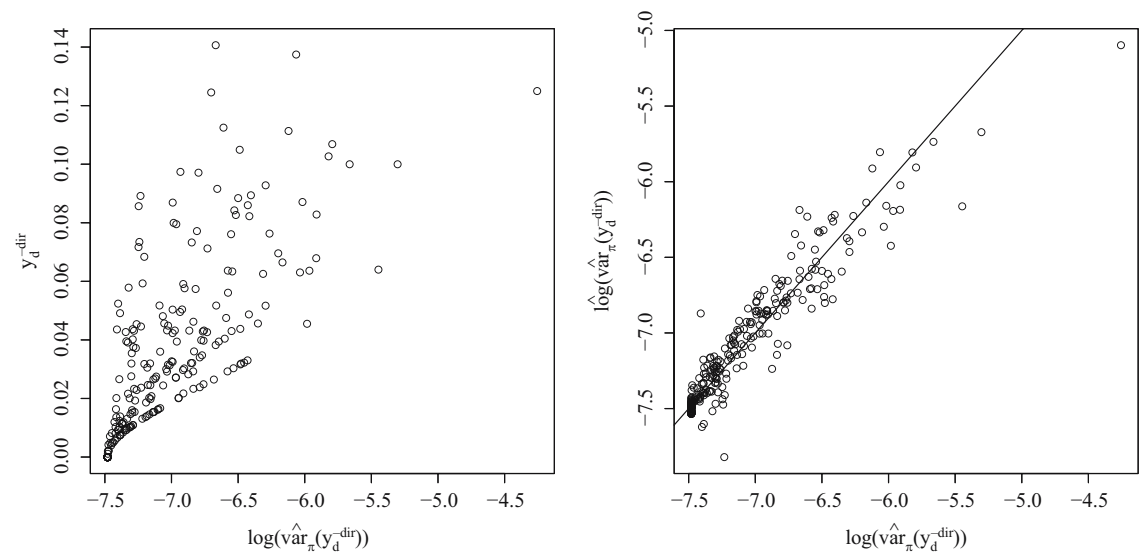

Fig. 2. Dispersion graphs for observed values under the fitted GVF model. 
Akaike Information Criterium (Akaike, 1974) in which the final model includes all the variables proposed in Equation (12). Table 1 presents the estimates of the regression parameters of the selected GVF model and the corresponding $95 \%$ confidence intervals. They are all significant with $p$-values lower than 0.001 .

The signs of the regression parameters basically say that the variance of the direct estimator of the PHMLAs has a non-linear increase with increasing $\hat{\bar{Y}}_{d}^{\text {dir }}$ and with decreasing $n_{d}$. This is intuitively expected. It is interesting to observe that $\beta_{6}^{v}<0$, so that the direct estimator and the sample sizes interact negatively. Figure 1 plots the residuals of Equation (5) with the explicative variables and parameters in Equation (1). The residuals do not present problems of heteroscedasticity or normality and no significant outliers are observed.

Figure 2 (left) plots a dispersion graph between the direct estimates and the logarithms of their estimated variances. Figure 2 (right) plots a dispersion graph between the predicted logarithms of the estimated variances and the logarithms of the estimated variances; that is to say, between the predicted and observed values of the GVF model.

In both cases, we observe a positive linear relationship that validates the selected GVF model. To construct Figure 3 (left), we partition the set of sample sizes into four subsets using the quartiles as cut points. More concretely, the first subset contains the smallest sample sizes and the fourth subset contains the greatest ones. From smallest to largest sample sizes from each subset are denoted by $n_{d}, 1 ; \ldots, n_{d}, 4$, where $d$ varies in the

Table 1. Estimated parameters of the GVF model.

\begin{tabular}{lrrrrrr}
\hline var & $\hat{\beta}$ & estimate & std.error & \multicolumn{1}{c}{$t$-value } & $p$-value & \multicolumn{1}{c}{ Conf.I 95\% } \\
\hline 1 & $\hat{\beta}_{1}^{v}$ & -7.3518 & 0.0344 & -213.94 & $<2 \times e^{-16}$ & {$[-7.419,-7.284]$} \\
$\hat{\bar{Y}}_{d}^{d i r}$ & $\hat{\beta}_{2}^{v}$ & 6.3722 & 0.9335 & 6.83 & $5.54 \times e^{-11}$ & {$[4.542,8.202]$} \\
$(\hat{\bar{Y}} d i r)^{1 / 2}$ & $\hat{\beta}_{3}^{v}$ & 5.2049 & 0.2898 & 17.96 & $<2 \times e^{-16}$ & {$[4.637,5.773]$} \\
$n_{d}$ & $\hat{\beta}_{4}^{v}$ & 0.0014 & 0.0001 & 14.47 & $<2 \times e^{-16}$ & {$[0.001,0.002]$} \\
$n_{d}^{1 / 2}$ & $\hat{\beta}_{5}^{v}$ & -0.0325 & 0.0045 & -7.17 & $6.94 \times e^{-12}$ & {$[-0.041,-0.024]$} \\
$\left(\hat{\bar{Y}}_{d}^{d i r}\right)^{1 / 2}$ & $\hat{\beta}_{6}^{v}$ & -0.3017 & 0.0196 & -15.40 & $<2 \times e^{-16}$ & {$[-0.340,-0.263]$} \\
\hline
\end{tabular}
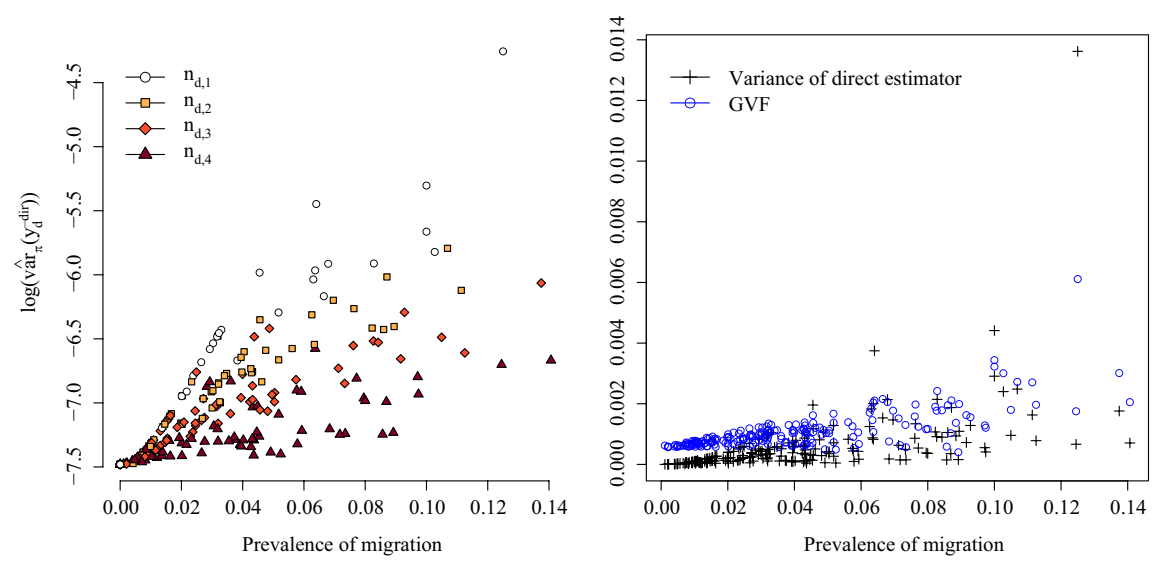

Fig. 3. Dispersion graphs for PHMLAs under the fitted GVF model. 
corresponding subset of municipalities. Figure 3 (left) illustrates how the log-variance of the direct estimator decreases when the sample size increases, which is natural from a practical point of view. In Figure 3 (right), it is possible to observe how the variance of the direct estimator and its prediction increase with similar behavior for different values in the estimation of PHMLAs.

Because of the results of the diagnosis analysis, we take the predicted GVF variances as true error variances for the Fay-Herrot model on the direct estimates of PHMLAs. In this case there is no need to apply a nonparametric variance estimation procedure like the one suggested by González-Manteiga at al. (2010).

\subsection{EBLUPs of International Migration Indicators}

This section fits a Fay-Herriot model to the estimates of the PHMLAs in municipalities of Colombia, performs a diagnosis analysis and presents the results of the employed methodology. For the Fay-Herriot model introduced in Equation (2), the use of covariates that provide the greatest possible degree of information at the municipal level is highly important. One of the objectives of this work is to estimate the target indicator in intercensal periods. For this reason, we use data from the census carried out right before the survey DHS2015 to introduce the auxiliary variables of the selected Fay-Herriot model. The first covariate, $x_{1}$, is the small area average of the unit-level variable

$$
x_{1 j}=\left\{\begin{array}{l}
1 \text { if any usual member of the household } j \text { lived abroad during } 2005, \\
0 \text { if there is no member of the household } j \text { who lived abroad during 2005, }
\end{array}\right.
$$

for $j=1, \ldots, N_{d}$. This variable is taken from the 2005 census. The second covariable, $x_{2}$, is the multidimensional poverty index described by Anand and Sen (1997) and is expressed as a percentage. This variable is calculated using the methodology provided by the Oxford Poverty and Human Development Initiative (OPHI) and is constructed with variables obtained from the 2005 Census.

The Fay-Herriot model that includes only the variable $x_{2}$ has $\mathrm{AIC}=-1,252.266$ and the model with the variables $x_{1}$ and $x_{2}$ has $\mathrm{AIC}=-1,311.098$. The second model has lower AIC, a better interpretation of the results and an acceptable analysis of residuals. For these reasons, the second model is selected. Tables 2 and 3 present the frequentist and Bayesian estimated parameters of the selected model.

Table 2 shows how the parameters are significant with $p$-values lower than 0.1 and gives the $90 \%$ confidence intervals. The negative sign of $\hat{\beta}_{1}$ indicates that as the PHMLAs increase in 2005, the PHMLAs decrease in 2015. The positive sign of $\hat{\beta}_{2}$ implies that municipalities with a higher poverty index have greater PHMLAs. Although we use a threshold of 0.1 we remark that for a threshold of $0.05, \beta_{1}$ is not significant. However,

Table 2. Estimated parameters of the selected Fay-Herriot model (frequentist).

\begin{tabular}{lrrrrcrr}
\hline var & $\hat{\beta}$ & estimate & std.error & $t$-value & $p$-value & \multicolumn{1}{c}{ Conf.i 90\% } & \multicolumn{1}{c}{ Conf.i 95\% } \\
\hline 1 & $\hat{\beta}_{0}$ & 0.020 & 0.006 & 3.102 & $1.91 \times e^{-03}$ & {$[0.009,0.031]$} & {$[0.007,0.033]$} \\
$x_{1}$ & $\hat{\beta}_{1}$ & -0.016 & 0.009 & -1.807 & $7.06 \times e^{-02}$ & {$[-0.031,-0.001]$} & {$[-0.034,0.001]$} \\
$x_{2}$ & $\hat{\beta}_{2}$ & 1.629 & 0.208 & -1.807 & $6.21 \times e^{-15}$ & {$[1.284,1.974]$} & {$[1.220,2.038]$} \\
\hline
\end{tabular}


Table 3. Estimated parameters of the selected Fay-Herriot model (Bayesian).

\begin{tabular}{llrcr}
\hline var & $\hat{\beta}$ & estimate & stand.error & \multicolumn{1}{c}{ Cred.i (95\%) } \\
\hline 1 & $\hat{\beta}_{0}$ & 0.022 & 0.005 & {$[0.011,0.033]$} \\
$x_{1}$ & $\hat{\beta}_{1}$ & -0.021 & 0.007 & {$[-0.033,-0.007]$} \\
$x_{2}$ & $\hat{\beta}_{2}$ & 1.625 & 0.183 & {$[1.258,1.948]$} \\
\hline
\end{tabular}

Table 3 gives the $95 \%$ credible intervals, showing that all regression parameters are significant under the Bayesian approach.

The residuals of the Fay-Herriot model are computed as the differences between the direct and EBLUP estimators, that is, $\hat{e}_{d}=\hat{\bar{Y}}_{d}^{d i r}-\hat{\bar{Y}}_{d}^{F H}, d=1, \ldots, D$. Figure 4 (left) shows how the residuals of the Fay-Herriot model are random without any evident correlation between municipalities. Figure 4 (right) illustrates how the randomness of residuals is maintained through the EBLUPs of the indicators.

Figure 5 presents dispersion graph of EBLUP and HB predictions versus direct estimates of PHMLAs. The model-based predictors provide more conservative and smooth estimations than those obtained using the direct estimators. The EBLUPs of PHMLAs are comparable to the direct estimates, but they have smaller mean squared errors, as shown in Table 4. Figure 6 illustrates how the EBLUP and HB estimates lead to an important reduction in the estimated coefficients of variation (ecv), particularly in areas with smaller sample sizes. The ratios between the estimated coefficients of variation of $\mathrm{HB}$ predictors and direct estimators are lower than one in $98 \%$ of the small areas. The ratios of ecv between HB and EBLUP predictors are lower than one in $42 \%$ of the small areas where $n_{d}<100$ and are lower than one in $40 \%$ of the considered small areas.

Table 4 presents the estimates of PHMLAs at the municipal level for large capital cities and border areas. These are the municipalities where the flow of migrants is of greater interest for studying the population dynamics in Colombia. Columns 2 and 4 contain the direct and EBLUP estimates, respectively. Columns 3 and 5 contain the estimated coefficients of variation for direct estimators and EBLUPs, respectively. Table 4 shows that EBLUPs have lower estimated coefficients of variation than direct estimators in all cases. Furthermore, the estimated coefficients of variation are lower than $20 \%$. This has considerable practical implications for the final publication of results and for decision making.

Table 4. Estimates of PHMLAs at the municipal level.

\begin{tabular}{lcccccr}
\hline Municipality & DIR & ecv-DIR & EBLUP & ecv-EBLUP & HB & ecv-HB \\
\hline Bogotá D.C. & 0.04359 & 14.75 & 0.03469 & 13.22 & 0.03453 & 15.07 \\
Cali & 0.08919 & 14.11 & 0.06040 & 10.53 & 0.06067 & 9.82 \\
Armenia & 0.08568 & 14.23 & 0.07397 & 9.57 & 0.07345 & 9.60 \\
Cartagena de Indias & 0.07348 & 16.80 & 0.03001 & 14.47 & 0.03078 & 14.27 \\
Soledad & 0.04368 & 24.79 & 0.02408 & 16.74 & 0.02437 & 17.32 \\
Chía & 0.04563 & 75.30 & 0.03563 & 13.87 & 0.03542 & 16.08 \\
Villa del Rosario & 0.04623 & 48.87 & 0.03362 & 9.82 & 0.03359 & 12.36 \\
Manaure & 0.04320 & 50.62 & 0.02751 & 17.29 & 0.02773 & 18.54 \\
Valle del Guamuez & 0.04872 & 67.01 & 0.02579 & 16.69 & 0.02609 & 18.50 \\
San andrés de Tumaco & 0.03600 & 45.94 & 0.01888 & 20.08 & 0.01922 & 22.29 \\
\hline
\end{tabular}



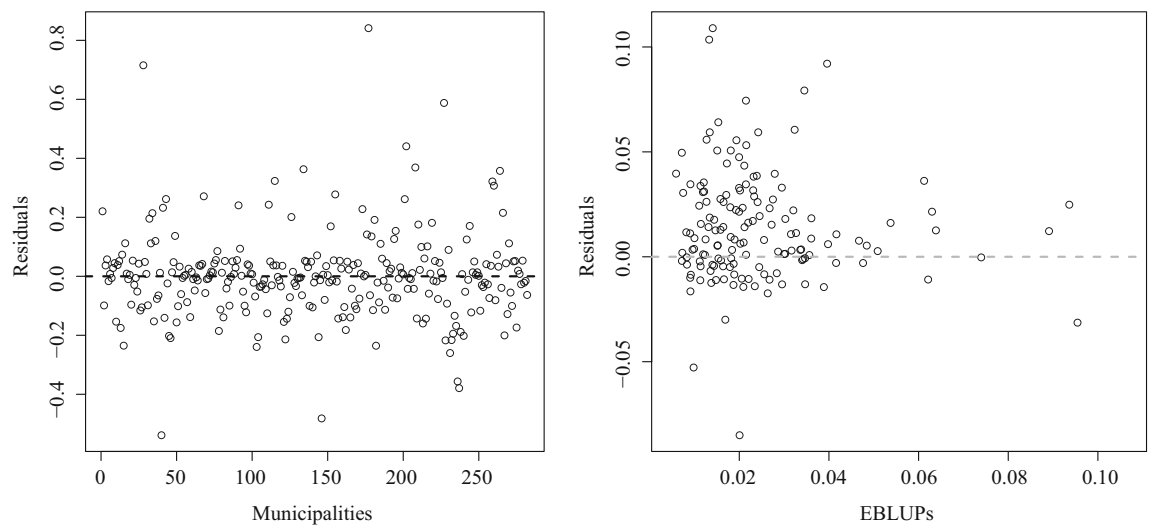

Fig. 4. Residuals of the selected Fay-Herriot model.
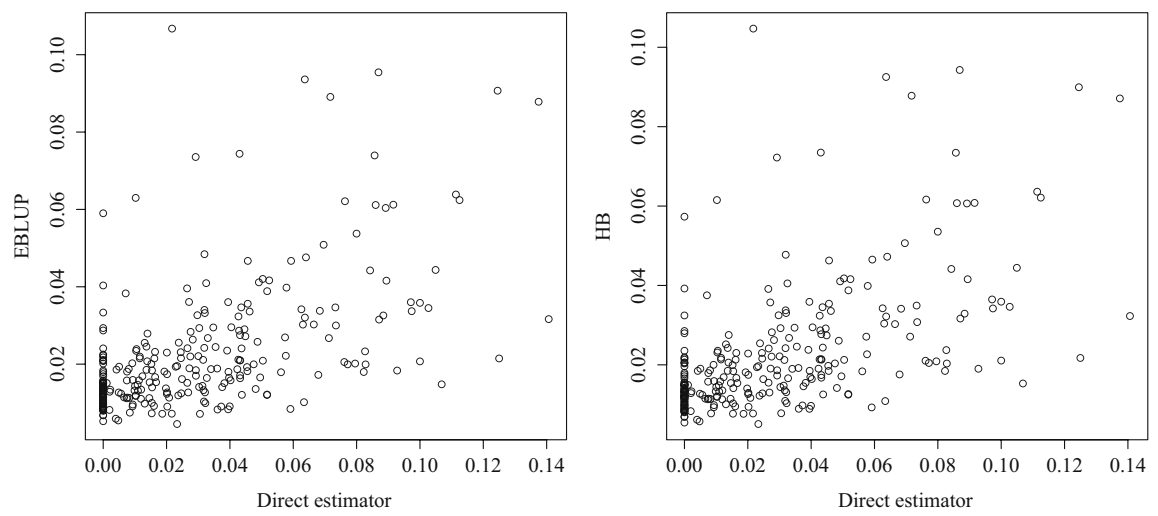

Fig. 5. EBLUP and HB predictions versus direct estimates of PHMLAs.
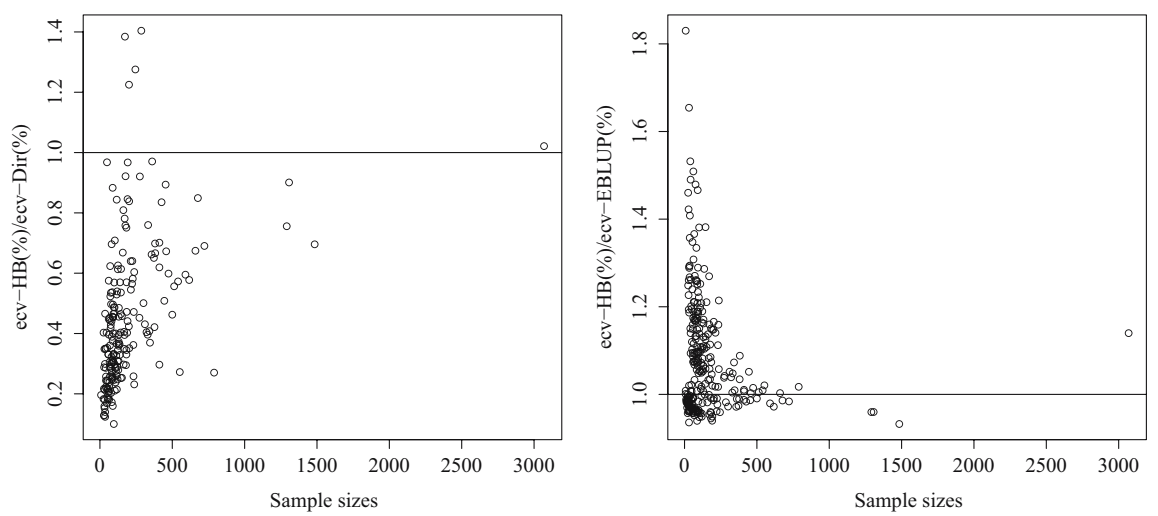

Fig. 6. Ratios HB/DIR (left) and HB/EBLUP (right) of estimated coefficients of variations versus sample sizes. 
Figures 7, 8 and 9 present the results for each geographic area and illustrate how in large capital cities and border zones the phenomenon of migration abroad is greater, which is consistent with population dynamics in Colombia. As we discussed, lower estimated coefficients of variation are obtained with the EBLUP and HB predictors. Figure 10 plots the predictions of model residuals (left) and random effects (right) under the Bayesian hierarchical approach. This figure shows some spatial patterns related to the large capital cities and border zones that could be investigated for incorporating additional information in the estimation process. As Colombia has more than 1,000 municipalities, constructing a proper spatial weight matrix is not an easy task. The color pattern might help to propose neighbor or proximity matrices depending on the similarity of color intensities, and later to

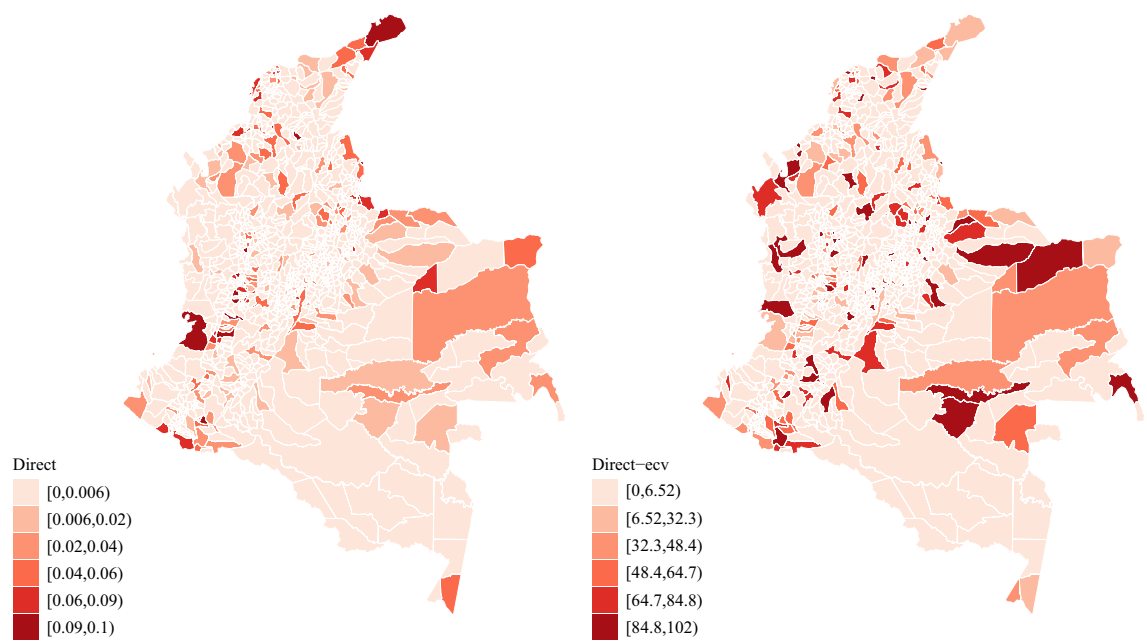

Fig. 7. Direct estimates of PHMLAs (left) and estimated coefficients of variation in percentage (right).

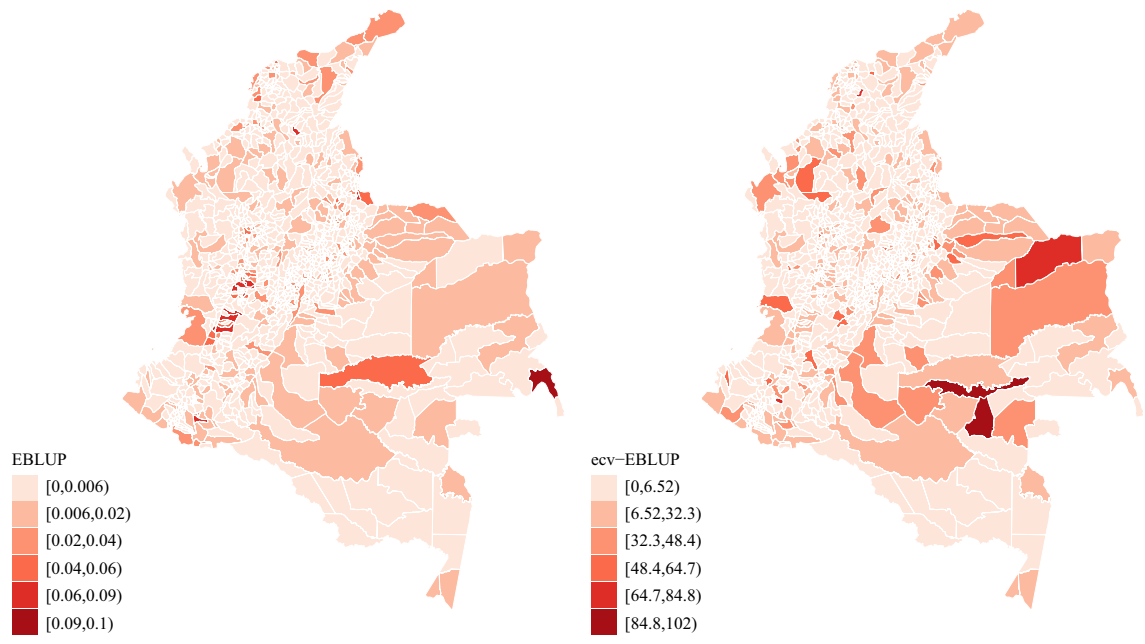

Fig. 8. EBLUP estimates of PHMLAs (left) and estimated coefficients of variation in percentage (right). 


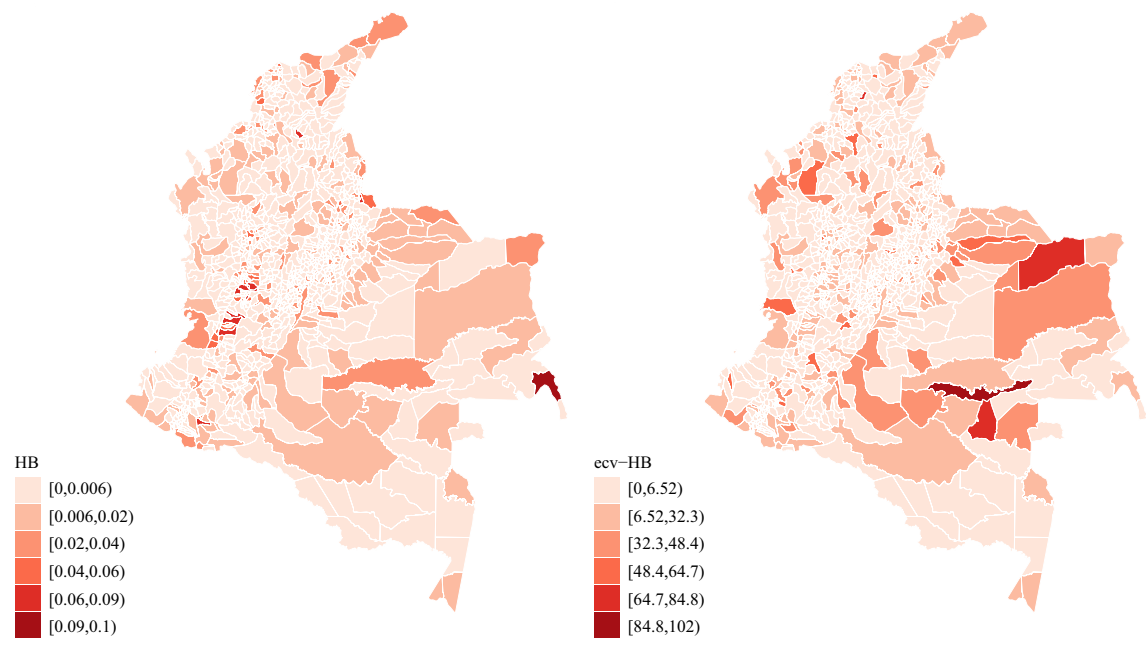

Fig. 9. HB estimates of PHMLAs (left) and estimated coefficients of variation in percentage (right).

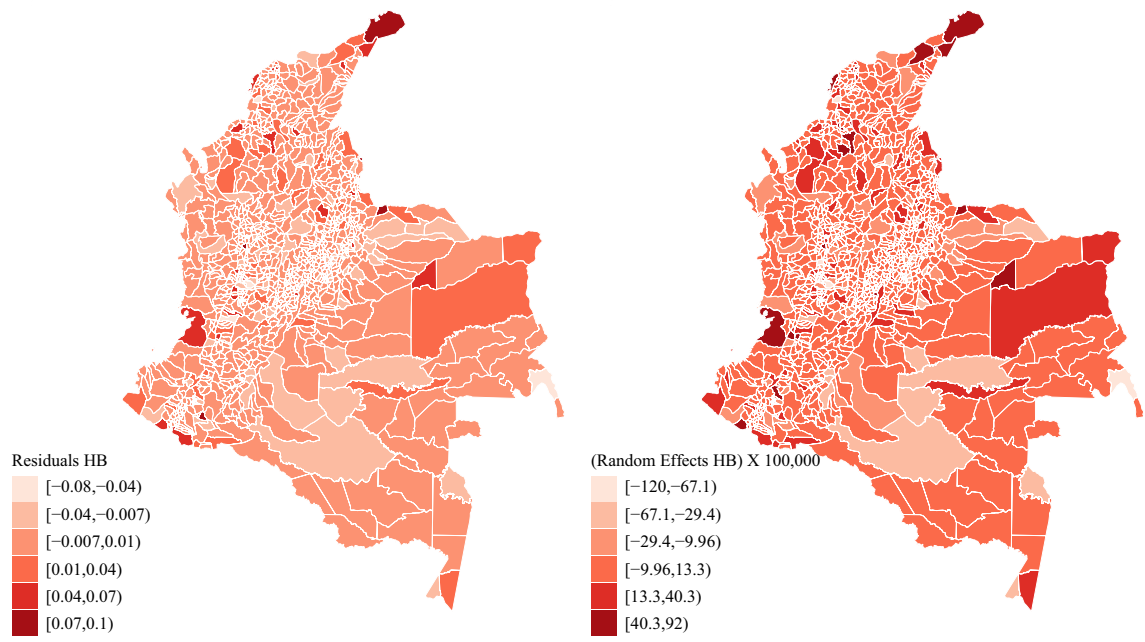

Fig. 10. Residuals (left) and (random effects) $x$ 100, 000 (right) for the HB approach.

fit a spatial Fay-Herriot model as it was done by Pratesi and Salvati (2008), Molina et al. (2009) or Marhuenda et al. (2013). This should be done for different spatial weight matrices until finding the right one that better captures the spatial dependence that it is not taken into account by the auxiliary variables. This is a multidisciplinary research project with its own entity and practical interest to be addressed in the future.

\section{Concluding Remarks}

The main Colombian cities, which are Bogota, Cali, Medellin, Barranquilla and

Cartagena, present some of the highest estimations in the proportions of households that 
have experienced international migration (with household members abroad). This is interesting inasmuch as these cities are the main areas where services, education and work are concentrated. It is likely that for many international migrants passing through, capital cities represent an intermediate phase to look for opportunities to emigrate, whereas for those who are from those municipalities it may be an attractive option to complement their educational and career projects or to search for a better quality of life. Moreover, high indicators can also be seen in municipalities of the coffee-growing region, which doubtless have a series of migration determinants and are different to those of the capital cities. This is a topic that merits further investigation in greater detail in order to better identify and characterize these differences.

Likewise, it must be highlighted that events that occur in specific geographical locations, in this case capital cities, often have consequences on their direct neighbors and the adjoining municipalities. In this sense, what was mentioned earlier can be reconfirmed: migratory phenomena do not occur homogeneously within the country. Instead, there is a selection filter meaning that there is a greater international emigration in some populations; for example, educational level and employment on an individual scale, but also at the scale of small areas. In fact, it is for these very same reasons that migration is affected by the economic cycle of countries and regions. Situations of economic crisis, such as the current situation in Venezuela, determine changes in the volumes of emigrants and immigrants, taking into account that individuals and families tend to look for the best opportunities in the areas identified.

The result obtained is also relevant insofar as, in Colombia, high-quality disaggregated indicators on a municipal level are only available after each population census, that is, every 10 or 15 years. In the intervening years, it is normal for changes to occur in migration patterns and in the intensity of the phenomenon. For this reason, sample surveys provide data that makes it possible to obtain estimates for interim periods (intercensal estimations) and for periods following censuses, whereby survey results are available (postcensal periods). Additionally, the possibility of including variables that could be catalogued as migration determinants in the models had not been addressed in other demographic sources and applications consulted in the DANE. Hence, it can be affirmed that small areas estimation is very useful for demographic analysis, since estimations that are more or less robust and very detailed can be obtained without needing to increase sample sizes.

The estimation of PHMLAs in Colombian municipalities in intercensal periods is of great importance due to the variability of the migratory flow over recent years. Direct estimations using surveys such as DHS make it possible to obtain estimates at the municipal level, with the disadvantage that these estimates display considerable sample errors. Some complex model-based approaches are not directly applicable to the Colombian case because they require rich data sources and are not designed to solve the problem of having small sample sizes in municipalities. Therefore, for the first time, a SAE procedure is conducted to estimate international emigration at the municipal level. More concretely, the estimations were based on the Fay-Herriot area-level model. The proposed statistical methodology can be easily applied to developing countries, where sufficient data sources are not available in quality and quantity. 
In order to estimate PHMLAs in intercensal periods, auxiliary variables at the municipal level were used. They were obtained from previous census studies and applied to a FayHerriot model and to the appropriate modeling of variance at the municipal level. By applying SAE methods, PHMLAs were obtained with estimated coefficients of variation lower than $20 \%$, enabling the results to be applied in statistical reports for decision making. The migration estimations are consistent with the population dynamics of Colombia, and they are similar to estimates achieved using the direct estimator, although with a considerably lower estimation error. The proposed methodology can be considered for the estimation of other indicators in future work. At this point, it is necessary to recognize that one limitation of the adopted approach is that it might lead to an underestimation of the real intensity of emigration by not counting cases of entire households that have moved away, as well as households that were dissolved through a separation or divorce where it is unlikely that the person who styed in Colombia reports the information related to the person who lives abroad. However, we believe that this does not affect, in a significant way, the main patterns that we can identify through the proposed estimation procedure. In any case, the alternative approaches that we examined do not produce estimations of the main small areas from which international emigration originates. Likewise, we showed that the proposed model has great potential for application in situations where there are indicators based on a last population census that are not updated. We have updated estimates based on sample surveys that, in principle, are not designed to give representative results at the local level, only at the regional level.

For the future, we could consider testing the statistical methodologies for estimating demographic quantities. The idea could be to construct artificial dynamic populations and conduct simulation experiments by randomly generating migrations and by extracting surveys from the population. The theoretical experiment should mimic the real data case under study and could give some insights about the proposed statistical methodology. In a different context, where the target was the estimation of small area poverty proportions, Marhuenda et al. (2017) and Morales et al. (2018) carried out this type of simulation experiments. They took into account the sampling-design distribution, but they worked in a non-dynamic scenario. The research target would be to transfer this idea to problems of demography.

On the other hand, we can test the methodology in a situation where you need not only the proportion of usual household members living abroad, but also the composition by age and sex of the international emigrants or an estimate of the main destinations of the emigrants. In this sense, the proposed methodology is not restricted to haveing the same categories of analysis in the origin and in the destination of the migration. The methodology can be used in cases when you need an estimation based on many small areas of one country or region, and you have only one destination of interest: the rest of the world, or several places of interest at a national level; for instance, the main five countries of destination.

\section{References}

Akaike, H., 1974. "A new look at the statistical model identification." IEEE transactions on automatic control, 19 (6): 716-723. DOI: https://doi.org/10.1109/TAC.1974.1100705. 
Anand. S., and A. Sen. 1997. Concepts or human development and poverty: a multidimensional perspective. United Nations Development Programme, Poverty and human development: Human development papers: 1-20. Available at: http://clasarchive.berkeley.edu/Academics/courses/center/fall2007/sehnbruch/UNDP\%20Anand\%20and\%20Sen\%20Concepts\%20of\%20HD\%201997.pdf (accessed April 2020).

Benavent, R., and D. Morales. 2016. "Multivariate Fay-Herriot models for small area estimation." Computational Statistics and Data Analysis, 94: 372-390. DOI: https:// doi.org/10.1016/j.csda.2015.07.013.

Burgard, J.P., M.D. Esteban, D. Morales, and A. Pérez. 2020a. "A Fay-Herriot model when auxiliary variables are measured with error." TEST, 29 (1): 166-195. DOI: https://doi.org/10.1007/s11749-019-00649-3.

Burgard, J.P., M.D. Esteban, D. Morales, and A. Pérez. 2020a. "Small area estimation under a measurement error bivariate Fay-Herriot model." Statistical Methods and Applications. DOI: https://doi.org/10.1007/s10260-020-00515-9.

Datta, G.S., M. Ghosh, R. Steorts, and J. Maples. 2011. "Bayesian benchmarking with applications to small area estimation.” Test, 20 (3): 574-588. DOI: https://doi.org/10. 1007/s11749-010-0218-y.

Datta, G.S., and P. Lahiri. 2000. "A unified measure of uncertainty of estimated best linear unbiased predictors in small area estimation problems.” Statistica Sinica, 10: 613-627. Available at: https://www.jstor.org/stable/24306735.

Edmonston, B., and M. Michalowski. 2004. "International Migration.” In: The Methods and Materials of Demography. Edited by H. Shryock, J. Siegel, and D. Swanson. Second Edition. New York: Academic Press.

Esteban, M.D., D. Morales, A. Pérez, and L. Santamaría. 2012. "Small area estimation of poverty proportions under area-level time models." Computational Statistics and Data Analysis, 56: 2840-2855.

Fay, R.E., and R.A. Herriot. 1979. "Estimates of income for small places: an application of James-Stein procedures to census data." Journal of the American Statistical Association, 74: 269-277.

González-Manteiga, W., M.J. Lombardía, I. Molina, D. Morales, and L. Santamaría. 2010. "Small area estimation under Fay-Herriot models with nonparametric estimation of heteroscedasticity." Statistical Modelling, 10(2): 215-239.

Hájek, J. 1971. Comment on "An Essay on the Logical Foundations of Survey Sampling, Part One.” In: The Foundations of Survey Sampling., edited by V.P. Godambe, and D.A. Sprott: 236. Holt, Rinehart, and Winston.

Hobza, T., and D. Morales. 2016. "Empirical Best Prediction Under Unit-Level Logit Mixed Models.” Journal of Offcial Statistics, 32 (3): 661 -69. DOI: http://dx.doi.org/10. 1515/JOS-2016-0034.

Hobza, T., D. Morales, and L. Santamaría, 2018. "Small area estimation of poverty proportions under unit-level temporal binomial-logit mixed models." TEST, 27 (2): $270-294$.

Jiang, J. 2007. Linear and generalized linear mixed models and their applications. Springer Verlag. 
Marhuenda, Y., I. Molina, and D Morales. 2013. "Small area estimation with spatiotemporal Fay-Herriot models." Computational Statistics and Data Analysis, 58: 308-325. DOI: https://doi.org/10.1016/j.csda.2012.09.002.

Marhuenda, Y., I. Molina, D. Morales, and J.N.K. Rao. 2017. "Poverty mapping in small areas under a two-fold nested error regression model." Journal of the Royal Statistical Society, series A. 180 (4): 1111-1136.

Migración-Colombia. 2015. Ministerio de Relaciones Exteriores: Comportamiento migratorio colombiano. Boletín anual de estadísticas. Enero a diciembre 2015. Migración.: 16-23. Availablet at: http://migracioncolombia.gov.co/index.php/es/.

Molina, I., N. Salvati, and M. Pratesi. 2009. "Bootstrap for estimating the MSE of the spatial EBLUP.” Computational Statistics, 24: 441-458.

Morales, D., M.C. Pagliarella, and R. Salvatore. 2015. "Small area estimation of poverty indicators under partitioned area-level time models." SORT-Statistics and Operations Research Transactions, 39 (1): 19-34. Available at: https://raco.cat/index.php/SORT/article/view/294361 (accessed August 2021).

Morales, D., M.M. Rueda, and M.D. Esteban. 2018. "Model-assisted estimation of small area poverty measures: an application within the Valencia Region in Spain." Social Indicators Research. 138 (3): 873-900. DOI: https://doi.org/10.1007/s11205-017-1678-1.

Plane, D.A. 1982. "An Information Theoretic Approach to the Estimation of Migration Flows.” Journal of Regional Science, 22: 441-456.

Prasad, N.G.N., and J.N.K. Rao. 1990. "The estimation of the mean squared error of smallarea estimators." Journal of the American Statistical Association, 85: 163-171. DOI: https://doi.org/10.2307/2289539.

Pratesi, M., and N. Salvati. 2008. "Small area estimation: the EBLUP estimator based on spatially correlated random area effects." Statistical Methods and Applications, 17: 113-141. DOI: https://doi.org/10.1007/s10260-007-0061-9.

Profamilia. 2015. Profamilia. 2015. Encuesta Nacional de Demografía y Salud - ENDS. Available at: https://profamilia.org.co/investigaciones/ends/.

Rao, J.N.K., and I. Molina. 2015. Small area estimation, second edition. John Wiley, Hoboken (NJ).

Raymer, J. 2007. "The Estimation of International Migration Flows: A General Technique Focused on the Origin-Destination Association Structure." Environment and Planning A, 39: 985-995. DOI: https://doi.org/10.1068/a38264.

Raymer, J., and A. Rogers. 2007. "Using Age and Spatial Flow Structures in the Indirect Estimation of Migration Streams.” Demography, 44: 199-223. DOI: https://doi.org/10. 1353/dem.2007.0016.

Raymer, J. 2010. "A general framework for estimating population movements." In Demographic aspects of migration: 73-96. DOI: https://doi.org/10.1007/978-3-53192563-9.

Schall, R. 1991. "Estimation in generalized linear models with random effects." Biometrika, 78, 4, 719-727. DOI: https://doi.org/10.2307/2336923.

Smith, P.W.F., J. Raymer, and C. Giulietti. 2010. "Combining available migration data in England to study economic activity flows over time." Journal of the Royal Statistical Society, series A, 173 (4): 733-753. DOI: https://doi.org/10.1111/j.1467-985X.2009. 00630.x. 
Smith, S.K., J. Tayman, and D.A. Swanson. 2013. A practitioner's guide to state and local population projections. Springer-Verlag.

Särndal, C.E., B. Swensson, and J. Wretman. 1992. Model assisted survey sampling. Springer-Verlag.

Willekens, F. 2008. "Models of Migration: Observations and Judgements." In International Migration in Europe: Data, Models and Estimates, 117-147, edited by J. Raymer, J. and Willekens, F. University of Southampton. Division of Social Statistics. John Wiley, Hoboken, New Jersey.

Received May 2019

Revised March 2020

Accepted May 2020 